\title{
Visualizing Recovery of Cognitive Function in Stroke
}

\author{
Andrea L. O. Hebb ${ }^{1 *}$, David Brandman ${ }^{1}$, Jai Shankar ${ }^{2}$, Adam O. Hebb ${ }^{3}$ \\ ${ }^{1}$ Division of Neurosurgery, Dalhousie University, QEII Halifax Infirmary Site, Halifax, Canada \\ ${ }^{2}$ Department of Diagnostic Imaging, QEII Hospital, Halifax, Canada \\ ${ }^{3}$ Colorado Brain \& Spine Institute, LLC, Englewood, USA \\ Email: andrea.hebb@cdha.nshealth.ca; ${ }^{*}$ alohebb@dal.ca; dbrandman@dal.ca; shivajai1@gmail.com; adam.hebb@aoh.md
}

Received October 7, 2013; revised November 15, 2013; accepted December 20, 2013

Copyright (C) 2013 Andrea L. O. Hebb et al. This is an open access article distributed under the Creative Commons Attribution License, which permits unrestricted use, distribution, and reproduction in any medium, provided the original work is properly cited.

\begin{abstract}
Hippocrates (460-377 BC) first described stroke over 2400 years ago. Stroke is the $4^{\text {th }}$ leading cause of death in Canada ( $3^{\text {rd }}$ in the USA) and the primary cause of permanent motor and cognitive disability. The majority of strokes are ischemic. The extent of cerebral dysfunction and thus the severity of stroke are based on the location, severity and duration of ischemia. Stroke management and prognosis encompass early recognition of the onset of stroke and post-stroke determination of the extent of brain injury aided by clinical stroke scores and diffusion-weighted imaging. Cognitive domains most likely to be affected following stroke are memory, orientation, language, attention and executive function. While the vast majority of functional recovery occurs within the first 3 months post-stroke, the neural mechanisms promoting recovery are not well understood. Investigations into the neural plasticity of brain areas after a lesion demonstrate that the adult brain can be shaped by environmental inputs, such as rehabilitation techniques. Many rehabilitation techniques are actively being pursued, including brain-computer interfaces providing sophisticated methods for detecting rehabilitation-associated changes in cerebral physiology. The success of such strategies visualized with functional magnetic resonance imaging and positron emission tomography may provide an objective complement to clinical evaluations.
\end{abstract}

Keywords: Ischemia; Magnetic Resonance Imaging; Positron Emission Tomography Rehabilitation; Brain-Computer Interface

\section{Introduction}

Stroke occurs following obstruction of blood flow and resultant neurological death to specific affected brain regions [1-4]. Stroke, or brain attack, is the $4^{\text {th }}$ leading cause of death in Canada ( $3^{\text {rd }}$ in the USA) and the primary cause of permanent motor disability. Each year in the United States approximately 700,000 people over the age of 18 experience a new or recurrent episode; on average, 1 person has a stroke every 45 seconds [5]. In Canada, the annual incidence of stroke is estimated to be 50,000 with one stroke occurring every 10 minutes. In children under the age of 19 years, for every 10,000 Canadian children, there are 6.7 strokes [1,6]. In Europe deaths occurring from stroke average about 650,000 per year [7]. Of every 100 persons that suffer a stroke, 15 will die, some fairly quickly and others later, 10 will return back to neurological baseline, 25 will recover with minimal disability, 40 will recover with moderate to se-

"Corresponding author. vere disability and 10 will become dependent and require long-term care. Depending on stroke etiology and age, a stroke survivor has a $20 \%$ chance of experiencing another stroke within 2 years. The average acute care stroke cost is approximately $\$ 30 \mathrm{~K}$ per patient, leading to an annual cost of about $\$ 2.7$ billion per year in Canada [8] and $\$ 64$ billion annually in the United States [5,9].

\section{Types of Stroke}

There are two broad categories of stroke: haemorrhagic and ischemic. The rupture of blood vessels and subsequent bleeding in or around the brain are classified as intracerebral and subarachnoid haemorrhagic strokes, respectively. Approximately $87 \%$ of strokes are ischemic, caused by inadequate brain circulation due to cerebral artery occlusion [2]. An ischaemic stroke may be caused by a large artery atherosclerosis (i.e. carotid stenosis), a cardioembolism or a small-artery occlusion. Up to $14 \%$ of all ischemic strokes occur in young adults ( $<50$ years 
of age) [10]. Cardiovascular risk factors, including hypertension, hypercholesterolemia, diabetes and smoking, pre-dispose patients to their life-time risk of developing an ischemic stroke [11,12]. There are 2 subcategories of ischemic stroke: thrombotic and embolic. Thrombotic stroke involves the narrowing and blockage of blood vessels secondary to arteriosclerosis while embolic stroke involves the blockage of a blood vessel secondary to a blood clot formed elsewhere in the body (usually the heart) that lodges in blood vessels in the brain [1,2]. The Trial of Org 10172 in Acute Stroke Treatment (TOAST) classifies ischemic stroke according to etiology into 5 categories: large-artery atherosclerosis, cardioembolic, small-vessel occlusion (e.g. lacunar infarct), stroke of other determined or undetermined etiology [13].

One of the most common stroke distributions follows from occlusion of the middle cerebral artery (MCA), the largest branch of the internal carotid. The MCA artery and its derivatives supply the majority of the outer surfaces of the brain, as well as a large component of the deeper grey and white matter structures, including the basal ganglia and internal capsule. Clinical symptoms concomitant with MCA occlusion depend on the size and location of the occlusion; these may include hemiparesis, hemiplegia, aphasia, dysarthria, dysphagia, visual field defects and visual-spatial neglect [14].

The anterior cerebral artery (ACA) branches upward and forward from the internal carotid artery and its distribution supplies most of the medial surface of the cerebral cortex, the frontal lobes, portions of the basal ganglia and anterior portions of the corpus callosum. Clinical symptoms concordant with ACA occlusion include cognitive dysfunction, changes in personality, and weakness, especially of the legs [15].

In most people, the posterior cerebral artery (PCA) stems from the basilar artery but origination from the ipsilateral internal carotid artery (fetal circulation) [16] occurs. The PCA supplies the midline temporal and occipital lobes bilaterally. Patients present with interesting and diverse spectrum of visual and sensory deficits and seldom motor deficits with $30 \%-70 \%$ of young stroke victims returning to work within 90 days [17].

Magnetic resonance imaging (MRI) is being increasingly used for anatomical imaging for acute stroke care while functional MRI (fMRI) and similar platforms are being used to visualize brain activation patterns in recovering stroke patients.

\section{Cognitive Deficits Following Stroke}

New cognitive deficits appear in approximately 30\% of stroke patients [18]. Cognitive domains affected following stroke include memory, orientation, language, atten- tion and executive function relative to age matched healthy controls $[19,20]$. Among young $(<50$ years) or old ( $>50$ years) adults processing speed, working memory, and attention were most commonly affected up to 11 years post-stroke $[10,21]$. Large vessel disease with strategic single and multiple infarcts and small vessel disease with progressive white matter lesions underlie the heterogeneity of vascular cognitive impairment presentation [22]. Cognitive impairment is most often coupled to infarcts localized in the left anterior and PCA territories post-stroke [20]. Stroke may impair cognition by injury to large regions of cerebral cortex, subcortical white matter connection fibers, or specific regions of the thalamus that are implicated in memory and attention [23].

Clinical symptoms associated with PCA occlusion depends on the location of the occlusion and resultant infarction, and may include thalamic syndromes, Weber's syndrome (characterized by the presence of an oculomotor nerve palsy and contralateral hemiparesis or hemiplegia), contralateral hemiplegia, hemianopsia and a variety of other symptoms, including color blindness, failure to see to-and-fro movements, verbal dyslexia, and hallucinations. The most common finding is occipital lobe infarction leading to an opposite visual field defect [24]. In particular, patients with left PCA lesions have defective mental visual imagery and such deficits stem from a loss of knowledge about the visual appearance (shape and color) of objects [25]. Patients that display cortical and deep infarcts (thalamic and/or mesencephalic involvement) also develop sensory (ventrolateral thalamic ischemia), and neuropsychological deficits including memory deficits, aphasia, neglect and anosognosia. Moreover, there was an increased risk of stroke recurrence and poor outcome in those patients with concurrent basilar artery disease (which gives rise to the PCA in most people) [16,26-28]. Some cortical lesions may be more devastating in older persons, and other lesions may surprisingly have little effect on cognitive and behavioral function [29]. After adjusting for age and physical impairment, cognitive impairment significantly decreased activities of daily living (ADL) and independent living [20].

Cerebral small vessel disease is a common cause of cognitive decline and vascular dementia characterized by executive/attentional dysfunction, but relatively intact episodic memory [30,31]. Lacunar strokes appear to be highly correlated with cognitive dysfunction in patients. Mild cognitive impairment superimposed on hippocampal atrophy translates to a severe, episodic memory disorder, while patients with mild cognitive impairment secondary to multiple subcortical infarcts display executive dysfunction [32]. In patients followed for 3 years post stroke, recurrent lacunar strokes and increased white 
matter hyperintensities on MRI were noted in 24\% of patients which correlated with subsequent reduced functional outcomes as measured by Katz's Index of ADL [33].

Diffuse periventricular hyperintensity as visualized on MRI following initial cerebral thrombosis is associated with the development of dementia and secondary complications of stroke including recurrence of stroke, pneumonia and motor disorders [34]. Combined occurrence of white matter lesions and lacunar infarctions decreased processing speed and memory to a greater extent relative to elderly individuals than each of the two single vascular brain lesion types alone [35]. Independent of cardiovascular risk factors, white matter lesions, and brain atrophy, non-demented persons having multiple infarcts perform poorer on tasks of memory, processing speed, and executive function, relative to study participants with no infarcts [36].

Diffusion-tensor MR imaging, a technique to quantify the white matter connectivity between brain regions that is damaged in subcortical infarctions, is best correlated with executive function and is sensitive to changes in brain structure that occur over time. MRI parameters can differentially correlate with cognitive domains in patients with symptomatic sporadic small vessel disease. For example executive dysfunction 1 year post-MRI was best predicted by diffusion tensor imaging (DTI) (fractional anisotropy), while DTI together with measures of brain volume, age, gender, and premorbid IQ, accounted for a large percentage $(74 \%)$ of the executive function score variance in cerebral small vessel disease [30]. Findings from MRI investigations may be employed in the determination of long-term prognosis of functional status following stroke.

\section{Implications for Rehabilitation Post-Stroke}

While the vast majority of functional recovery occurs within the first 3 months post-stroke, the neural mechanisms promoting recovery are not well understood. Diffusion (DWI) and perfusion-weighted (PWI) MRI are relatively new MR techniques increasingly used in acute stroke. DWI has a high sensitivity to shifts of water between extracellular and intracellular spaces depicting random movements of water protons which in ischemic brain tissue are restricted due to stroke-induced cytotoxic edema. Perfusion studies are based on the bulk flow of the blood (not on the oxygenation of the hemoglobin like Blood Oxygenation Level Dependent (BOLD)). In the first few hours post-stroke PWI provides metabolic and hemodynamic data of the brain, based on the movement of perfused contrast material. The difference between the diffusion and perfusion abnormalities provides a measure of the ischemic penumbra or the brain tissue at risk for infarction [37]. DWI and PWI have been employed both for the detection and characterization of ischemic insult before and following reperfusion techniques to break down an occlusive thrombus (i.e. administration of recombinant tissue plasminogen activator (rt-PA)) [38]. DWI and PWI will not illuminate the process of brain plasticity, but DTI or functional MRI may and as such may be used to tailor individual rehabilitation programs to impact clinical recovery. Theories and mechanisms of brain plasticity are vast. Proposed mechanisms of neuronal reorganization including recruitment of anatomically distinct but functionally homologous pathways, synaptogenesis, dendritic sprouting, and activation of previously existing but silent neural pathways would fall under the umbrella of brain plasticity [39]. A greater understanding of the underlying mechanisms of brain plasticity during recovery after stroke could potentially facilitate rehabilitation programs geared towards improving post-stroke motor, emotional and/or cognitive function.

\section{Use of fMRI to Correlate Brain Activity with Rehabilitation Techniques, Plasticity and Outcome}

Over several months following stroke, ischemic infarction results in brain tissue atrophy and neuronal cell death [40]. Yet throughout adulthood the brain is capable of plastic reorganization following infarction [41] which may occur spontaneously after stroke [42]. Stroke recovery involves the resolution of diaschisis and neural reorganization in undamaged brain tissue both ipsi- and contralesional [43]. Often newly relearned behaviors are represented diffusely over more cortical regions due to adaptive functional organization of perilesional brain areas as well as those connected to but remote from the infarct [44-46]. Improvement occurs usually within the first 3 - 6 months following stroke and is based on the location and size of infarction, repair of damaged tissue and cerebral reorganization, patient age and reduction of stroke risk factors, including control of hypertension, to evade future episodes [47]. Within one week after stroke onset $50 \%$ of the best relative recovery of brain activity occurs in individuals [48]. After a stroke, recovery that continues beyond 3 or 4 weeks has been ascribed to brain reorganization in the cerebral cortex, in which functions formerly executed by ischemic areas are performed by supplementary ipsilateral or homologous areas in contralateral brain regions. This forms part of an adapting distributed and cortical substitution functional neuronal network of uncrossed motor and sensory pathways for damaged pathways [41,47,49,50].

Following cerebral infarction, neural energy utilization and central reorganization can be measured using the fMRI BOLD technique [51,52]. BOLD is based on the change in oxygenation of the blood secondary to blood 
flow. Monitoring brain function may permit the evaluation of brain plasticity and serve as a tool to aid the development of novel validated task-specific clinical protocols. These protocols may optimize rehabilitation strategies improving patient rehabilitation outcomes and applied to individual cases of brain injury permit a peek into the brain during human thought processes $[38,51,52]$. At the very least, use of techniques such as fMRI will permit the identification of viable brain areas supporting neuronal plasticity and permit clinicians to synchronize functional training (i.e. motor, cognition and language) with predicted clinical recovery [53].

\section{Brain Reorganization and Recovery Following Stroke}

Clinical recovery and brain reorganization has been most commonly demonstrated with regards to motor and sensorimotor function following focal brain lesions in response to specific tasks or stimuli. Noninvasive techniques such as fMRI can map functionally relevant cerebral reorganization following resolution of edema and survival of the ischaemic penumbra [54-57]. Such network reorganization and functional recovery would be affected by the location of the infarct and residual functional anatomy surrounding the lesioned area. In addition recruitment of nonaffected regions of the functional network would be instrumental in promoting relevant adaptive changes that would facilitate recovery that has been observed over weeks, months, and now and again years following focal damage [44,56]. Studies illustrate robust reorganization in the activity patterns of specific brain networks with recovery from hemiplegic strokes although vast variability exists between patients in the pattern and stability of activity.

\subsection{Sensorimotor and Motor Recovery Following Stroke}

It is well documented that regions adjacent to damaged sensorimotor areas can progressively take over lost function previously ascribed to the lesioned area. Moreover, different cerebral mechanisms underlie complete, partial and poor functional recovery [39]. In early postinsult stages, unaffected perilesional tissue in cortical stroke and intact but deafferented cortex in subcortical strokes is recruited. This underlies recovery or partial gain of function. In some cases, activation of areas not normally engaged in such activities is evidence of compensatory recruitment to maintain task performance. For example, compensatory cognitive strategies (such as generating mental images) engage the dorsolateral prefrontal cortex or the superior parietal cortex in motor paralysis to promote motor function recovery [55,57]. Within the first 4 weeks post-stroke, integrity of de- scending connections from the ipsilesional cortico-spinal tract system is critical for motor recovery as revealed by single-pulse transcranial magnetic stimulation (TMS) [40]. Mechanisms underlying the dynamic reorganization of cerebral networks during recovery from ischaemic brain infarction can be visualized using fMRI and TMS, which can detect reorganization of sensorimotor pathways after stroke appearance [39]. With motor recovery over time, greater recruitment of the affected primary motor cortex (M1) and decreased activation of the unaffected M1 and supplementary motor area has been observed with fMRI [58]. Post-stroke improvements in arm and hand motor function involve reorganization in primary and secondary sensorimotor areas in both affected and non-affected hemispheres as revealed by changes in synaptic activity [59]. In hemiparetic stroke, recovery of finger movements was associated with activation of premotor cortical areas both ipsilateral and contralateral to the affected hand and enhanced perilesional infarct hypoperfusion and activation [40].

Immediately following stroke the dynamic and sustained process of brain recovery commences. In human ischemic brain disease the mechanisms underlying recovery can be studied using non-invasive techniques including functional neuroimaging and TMS. In the acute stage of stroke (within 24 hours of stroke) MRI identifies tissue that has potential for rescue through reperfusion techniques. In the subacute and chronic stages of stroke repetitive training, constraint induced training and imagery can facilitate motor recovery promoting a re-emerging activation in the affected hemisphere and functional deficit compensation assisted by local perilesional and large-scale bihemispheric circuits [49,60,61]. Correlations of motor function reappearance with specific brain regional networks outlined on fMRI must consider that the motor system is part of a larger system that is affected by expectation, observation, imagination and carrying out of movements [62]. Brain-computer interface with electroencephalography (EEG) is also being used to provide direct feedback on activation of alternate cortical areas to facilitate accessory connectivity.

Rehabilitation of hemiparesis caused by stroke would include activity-dependent adaptive neuronal plasticity induced by constraint-induced movement therapy (forces the use of the affected side by restraining the unaffected side) with the structural and functional changes in the brain detected with TMS, positron emission tomography (PET) and fMRI [63]. Motor hand function improved following motor rehabilitation techniques visualized as increased BOLD signal in the affected primary sensorimotor cortex following therapy. Where increased synaptic efficiency was not possible due to lesions in area M1 and descending pyramidal tracts, reorganization and recruitment of additional sensorimotor cortical areas was 
observed with restoration of behavioral function [64]. Patients with somatosensory lesions or ventroposterolateral thalamic infarcts demonstrated a decreased intracortical inhibition on the affected side. Patients with superior cerebellar artery territory infarcts demonstrated decreased intracortical facilitation and increased intracortical inhibition as measured by TMS and fMRI. Together, abnormal activation of motor and premotor cortical areas following stroke underlie deficits in voluntary motor control and these effects dissipate following 2 weeks of constraint-induced movement therapy indicative of modulation of neuroplastic mechanisms and traininginduced adaptive reorganization of the motor system $[54,65]$. Rehabilitation of motor function, recovery and resulting cortical reorganization brain activation patterns support use-dependent neuroplasticity and increased activation of marginally functional neurons as well as areas ipsilateral to a unilateral brain lesion. This emphasizes the importance of prompt intervention for stroke which tries to save penumbra tissue in the hyperacute stages. However, recovery success is also dependant on lesion volume, patient characteristics, intervention protocols, and outcome measures [66-70].

\subsection{Brain-Computer/Machine-Interface (BMI) Rehabilitation in Motor and Cognitive Systems}

Recent rehabilitation approaches to facilitate motor system recovery include transcranial magnetic low frequency stimulation of injured cortex paired with motor tasks and brain-computer/machine-interface (BMI) rehabilitation [71]. Such applications have been used in humans, rats and non-human primates. In clinical studies such paired interventions have the potential to increase upper extremity function 12 weeks post-rehabilitation and approximately 7 months post-stroke [66]. BMI technology is so exciting, because there is such an enormous need to introduce technology that will improve functional independence. Functional MRI permits direct study of training-induced plasticity accompanying functional recovery in stroke patients and conveys plastic changes in brain function $[54,55,57]$.

The utility of fMRI in the identification of reduced neural function in areas supporting cognitive dysfunction may identify patients who are at increased risk for developing dementia, and allow for the implementation of early cognitive rehabilitation techniques whose success could be further monitored using fMRI [72]. There are often no distinct lesions identified with conventional MRI or CT scans; for instance, lesions of the medial temporal lobe may be difficult to visualize [19,72]. In other cases, patients with left hemispheric, subcortical, and large lesions demonstrated deficits in episodic verbal memory as assessed by the Rey Auditory-Verbal Learn- ing Test [73]. Among non-aphasic stroke patients with cognitive deficits, verbal episodic memory deficits were related to predominantly left hemisphere lesions of medial temporal, thalamic, and frontal regions while an immediate recall deficit was observed with thalamic lesions and deficiency of cued-recall-frontal lesions [74]. However, within subregions of the medial temporal lobe there is a functional-anatomic dissociation for memory retrieval versus contextual memory that may only be revealed with fMRI region-of-interest (ROI) analysis of the hippocampus, parahippocampal and/or perirhinal cortex [75]. In elderly individuals with mild cognitive impairment, fMRI scanning revealed that those who showed cognitive decline over the subsequent 2.5 year clinical follow-up; a significantly greater extent of the right parahippocampal area was activated during encoding, despite equivalent memory performance [76]. This pattern of activation is thought to be attributed to compensatory recruitment needed to uphold task execution.

Reproducibility of fMRI activation during encoding tasks for word-pair, pattern, and scene encoding within a given ROI positioned within the medial temporal lobe, inferior frontal and fusiform gyri was good, showing very little inter and intra-subject variability [77]. In a dynamic visual-spatial imagery task reliability of brain activity in occipital, parietal, premotor and prefrontal regions of interest was measured for consistency across trials. Interestingly activation in the dorsolateral prefrontal cortex, an area involved in the processing and solving of cognitive tasks, decreased with increased task trials suggesting competency and learning in this task with repeated serial performance. However, it should be underscored that averaging across trials in fMRI analysis might result in erroneous interpretation dismissing this area as relevant for task processing and functional recovery [78]. The use of fROI can eliminate this error of data analysis in higher-order cognitive systems.

Post intervention fMRI may provide a cortical map in response to rehabilitation techniques focused on cognitive function. For example, silent ischemic lesions appear on MRI in otherwise healthy nondemented elderly individuals and as such represent a primary target for cognitive rehabilitation/training to prevent the onset of vascular dementia [79]. Even among adult patients aged 18 50 years with first-ever ischemic stroke, silent brain infarcts on MR imaging as defined as focal, multifocal, or confluent hyperintensities on T2-weighted sequence were predictive of recurrent stroke $[80,81]$.

\subsection{Attentional Networks}

Recovery of behavioral function following stroke whether the deficits are primarily motor or cognitive, relies on the integrity of the attention network which cannot be assessed without having the patient perform a perceptual, 
cognitive and/or motor task [82]. Attention training following stroke improved the allocation of attention and working memory demands in complex tasks and lowered patient frustration which translated to self-reported improvements of attention in situations of daily living and these effects persisted for 6 months [83]. It is yet to be determined the patient characteristics such as time postinjury, extent of brain injury, and age as well as initial levels of performance that direct as well as optimize the clinical outcomes of cognitive rehabilitation that result in clinically significant improvements in memory, visual processing, visuospatial attention, verbal reasoning and executive function [83-85]. The efficiency and independence of attentional networks as visualized by fMRI delineates separate but interlinking neuroanatomical pathways subserving each function: right hemispheric frontal and parietal regions related to alerting, superior parietal lobe controlling orienting functions while the anterior cingulate and lateral prefrontal cortex subserving executive function [86]. It is particularly obvious that the right hemisphere plays an important role in attention as hemineglect is seen primarily arising from damage to the right parietal lobe, specifically the inferior parietal lobule [82]. Ability to maintain attention to spatial locations, but not verbal items, even in simple detection tasks was impaired relative to healthy individuals and stroke control subjects [87]. Following right-hemisphere stroke maladaptive hyperexcitation of neural pathways in the undamaged left hemisphere exacerbates attention deficits [88]. Among patients with visuospatial hemineglect, enhanced alertness and a major improvement in neglect test battery performance was achieved following a 3 week computerized alertness training session as revealed by neuropsychological tests and fMRI. Amelioration of neglect was accompanied by increased activity in the frontal cortex, anterior cingulate cortex, precuneus, cuneus and angular gyrus of the right and left hemispheres, areas that have previously been linked with alertness and spatial attention $[84,89]$. With recovery from spatial neglect there is a general increase in activity of the fronto-parietal attentional network on the lesioned hemisphere with subsequent decreased activity in homologous left hemisphere regions indicative of neural reorganization [90]. Strategic cognitive interventions partially restored visuospatial attention, visuoperceptual and perceptuomotor function as well as visual memory and increased blood flow as established by PET that persisted 7 months following posterior cerebral artery distribution infarction [91].

Visuospatial attention training improved attention task performance (accuracy and reaction time) which was accompanied by alterations in activity in attention delineated pathways including increased activation in the anterior cingulate cortices and posteromedial portions of the parietal lobe with decreased demand on frontal lobe function. Functional restoration in the domains of attention, memory and executive function were associated with a redistribution of neural activities within frontal cortical networks with an overall lessening of diffuse cortical activity patterns indicative of more efficient processing [92]. Increased cognitive load may also improve motor rehabilitation. In patients with right MCA stroke anterior cingulate activity was increased in the nonparetic right hand following execution of movements requiring self-monitoring and presumably increased attentional demands relative to visually guided movements of the same motor task. Moreover, the lesioned hemisphere was more active on fMRI in stroke patients relative to healthy controls suggesting vast bihemispheric motor system reorganization following a focal lesion in the right hemisphere [93]. This displays different brain activation for self paced movements versus cued movements. The self paced movements require the medial frontal cortex (supplementary motor area), whereas the cued movements do not. In our experience when we resect the SMA, self directed movements are pretty much eliminated, but patients still have strength. However, it is not clear whether this would support increased cognitive load leading to improved motor rehabilitation.

Techniques such as fMRI and TMS can be employed to visualize cortical reorganization for cognitive processes and skilled movements induced by injury. These techniques can also optimize neurophysiological interventions enhancing activity-dependent synaptic plasticity to maximize recovery [94]. Functional MRI can delineate neural networks underlying specific cognitive and motor deficits and the unique pattern of activation specific to the individual in order to develop validated reliable optimal strategies for rehabilitation [59]. Increased activation patterns as revealed by fMRI in tests of memory and attention suggest that with varying damage (location and lesion size in various subcortical structures supporting attention and memory functions) an increased activation pattern is seen in additional neural structures as additional recruitment of neural areas occurs compensating for the damaged areas. Following rehabilitation interventions and the relearning of specific behaviors, fMRI may be utilized to identify the components of the newly functioning neural networks sustaining the behavior and distinguish between reactivation of previously damaged brain tissue and replacement of deficient tissue with intact systems [29].

\subsection{Language Rehabilitation}

The neural correlates underlying rehabilitative efforts to restore cognitive function are poorly elucidated [52, 95] although similar efforts to restore other cognitive functions, such as language may be applicable to these dis- 
cussions. Neuroimaging techniques have made a substantial contribution to the understanding of brain language function and neural networks affected in speechlanguage disorders following ischemic stroke [96].

Event related fMRI may use the BOLD contrast technique or perfusion-based fMRI which may be more accurate in representing functionality of brain regions at least in normal subjects performing various cognitive tasks. For example, perfusion-based fMRI is able to localize the region of neuronal activity more accurately than fMRI based on BOLD contrast techniques at least with respect in depicting activation in Broca's area during a verb generation task [97]. Notwithstanding, in two cases of Broca's aphasia, fMRI was performed prior to and following language training in patients with persistent and severe phonological anomia. Functional MRI was used to visualize brain activity patterns and cerebral reorganization coinciding with phonetic training-induced language improvement. In a patient with a small nonencompassing lesion in Broca's area, new language activation occurred in perilesional tissue after training while a patient with complete destruction of Broca's area showed activation in the right frontal region mirroring the left lesioned side [52]. In a separate study, Perani et al. [95] assessed the pattern of brain activity during letter and semantic-cued word retrieval in five aphasic stroke patients in an attempt to map brain activity underlying recovery following 6 months of language training. Similar to the study of Vitali et al. [52] the site and extent of the lesion and the amount of returned language function as assessed by task performance was associated with specific brain activation patterns. For example, good performance in word retrieval cued by letters activated Broca's area or the homologue in the right inferior frontal cortex, while deficits in semantic fluency which involve processes of semantic memory were defined by extensive patterns of cerebral activation reflecting an increased effort in retrieval, albeit unsuccessful [95]. The neural correlates of phonological cued language training were revealed with specific alterations in brain activation patterns, including perilesional reactivation and increased picture naming performance in chronic aphasics [52]. Currently the neural networks associated with improvement of cognitive function following language training in the damaged brain are not well publicized [52]. Yet recovery from aphasia involves changes in cognitive strategy and is mediated by the preserved perilesional neuronal networks and activation of homologous regions in the hemisphere contralateral to the infarct as revealed by fMRI [98]. In aphasic patients improved ability to name objects following intensive language training resulted in bilateral hemispheric recruitment of cortical activity [99]. In a heterogeneous sample of chronic aphasia patients fMRI was performed prior to and following overt naming performance language training. Language training increased brain activation in perilesional areas indicative of treatment-induced functional reintegration of damaged tissue as determined by ROI statistical analysis [100]. In aphasia, homologous areas in the right hemisphere were recruited one month post stroke followed by large left hemisphere perilesional areas 1 year post stroke [101].

As with motor recovery in patients with lesions to primary motor areas favorable recovery predominantly activates structures in the ipsilesional hemisphere. Overactivation of diffuse right hemisphere homologues may represent a maladaptive strategy which is reversed with rTMS contralateral hemisphere suppression and reintegration of left temporal areas into the functional language network [44]. Greater intensity of aphasia therapy as measured by increased number of hours per week over a shorter period of time produces favorable results while less intense therapy (reduced hours per week but more weeks) may offer little or even no benefit [102]. Although language function is predominantly a function of the left hemisphere; left inferior frontal (Broca's area) and superior temporal cortex (Wernicke's area), language recovery necessitates the restoration of the bilateral hemisphere language complex [103,104].

\subsection{Assessment of Successful Stroke Rehabilitation}

Functional MRI technology may be used to assess neuroplastic alterations in brain function by observing increased activation of regional cerebral blood flow to areas located ipsilateral to vascular lesions. To be sure increased activation would be associated with or facilitate recovery in clinical measures of motor, cognitive or language function following rehabilitation techniques in stroke [68]. Functional MRI will play an important role not only in the initial evaluation of patients following an acute ischemic event but also in the evaluation of rehabilitation techniques on behavioral function and resultant reorganization of neural pathways and restoration of cognitive function. Functional MRI studies may be used to help evaluate current treatment and rehabilitation strategies and test their efficacy in conditions such as stroke in which prognosis made solely on clinical data is difficult [105]. Increased cerebral blood flow following cognitive rehabilitation strategies restores function to underlying neuronal circuitry. Successful stroke rehabilitation requires the employment of current complementary technologies such as fMRI and PET in conjunction with clinical assessments for a complete understanding of the pathophysiological mechanisms underlying specific cognitive deficits in the acute and chronic stages of stroke.

\section{Conclusion}

The goal of rehabilitation is to reduce the physical and 
cognitive impairments and disabilities of patients with stroke and other neurologic diseases. Functional MRI permits the in vivo evaluation of regional brain function employing changes in regional cerebral blood flow as a putative surrogate marker for neural utility [106]. Neuroimaging may be used in conjunction with standardized neuropsychological testing in the study of single subject neuroplasticity following brain injury, synthesizing anatomical and behavioral skill learning. Neuroimaging would add an objective component outlining intensity and topography of neural activation patterns that occur within the context of predicted behavioral changes circumventing interpretation errors following rehabilitation efforts and inter-subject comparisons [107]. It should be underscored that in order to validate functional imaging (MRI or PET) as a clinical tool for the diagnosis, prognosis and management of pathophysiological conditions such as cognitive impairment following stroke, well controlled prospective studies will need to be performed [106]. Functional imaging may be used to assess changes in activation of brain regions after specific interventions directed at memory, attention, concentration, comprehension, insight, or other components of executive/cognitive function. Functional imaging may be used as a predictor of cognitive impairment to visualize the nature of the adaptive changes in cognitive neural networks and to delineate a specific patient's response to an intensive, multidisciplinary rehabilitation program.

\section{REFERENCES}

[1] Heart and Stroke Foundation of Canada, "Stroke statistics,” 2002.

http://www.heartandstroke.com/site/c.ikIQLcMWJtE/b.3 483991/k.34A8/Statistics.htm

[2] J. Jankowiak, “Brain Attack: Dial 91,” Neurology, Vol. 59, No. 10, 2002, pp. 10-11. http://dx.doi.org/10.1212/WNL.59.10.E10

[3] W. D. Smucker, J. A. Disabato and A. E. Krishen, "Systematic Approach to Diagnosis and Initial Management of Stroke,” American Family Physician, Vol. 52, No. 1, 1995, pp. 225-234.

[4] L. Snaphaan, M. Rijpkema, I. van Uden, G. Fernández and F. E. de Leeuw, "Reduced Medial Temporal Lobe Functionality in Stroke Patients: A Functional Magnetic Resonance Imaging Study,” Brain, Vol. 132, No. 7, pp. 1882-1888.

[5] W. Rosamond, K. Flegal, G. Friday, K. Furie, A. Go, K. Greenlund, N. Haase, M. Ho, V. Howard, B. Kissela, S. Kittner, D. Lloyd-Jones, M. McDermott, J. Meigs, C. Moy, G. Nichol, C. J. O’Donnell, V. Roger, J. Rumsfeld, P. Sorlie, J. Steinberger, T. Thom, S. Wasserthiel-Smoller and Y. Hong for the American Heart Association Statistics Committee and Stroke Statistics Subcommittee, "Heart Disease and Stroke Statistics-2007 Update,” Circulation, Vol. 115, 2007, pp. E69-E171.

http://dx.doi.org/10.1161/CIRCULATIONAHA.106.1799
18

[6] Public Health Agency of Canada, “Tracking Heart Disease and Stroke in Canada-Stroke Highlights,” 2011. http://www.phac-aspc.gc.ca/cd-mc/cvd-mcv/sh-fs-2011/in dex-eng.php

[7] World Health Organization, "World Health Report," 2007. http://www.who.int/whr/2007/whr07_en.pdf

[8] Statistics Canada, "Canada Health Measures SurveyCholesterol Levels of Canadians, 2009-2011,” 2012. http://www.statcan.gc.ca/pub/82-625-x/2012001/article/1 1732-eng.htm

[9] D. Lloyd-Jones, R. Adams, M. Carnethon, G. De Simone, T.B. Ferguson, K. Flegal, E. Ford, K. Furie, A. Go, K. Greenlund, N. Haase, S. Hailpern, M. Ho, V. Howard, B. Kissela, S. Kittner, D. Lackland, L. Lisabeth, A. Marelli, M. McDermott, J. Meigs, D. Mozaffarian, G. Nichol, C. O'Donnell, V. Roger, W. Rosamond, R. Sacco, P. Sorlie, R. Stafford, J. Steinberger, T. Thom, S. WasserthielSmoller, N. Wong, J. Wylie-Rosett, Y. Hong, American Heart Association Statistics Committee and Stroke Statistics Subcommittee, "Heart Disease and Stroke Statistics-2009 Update: A Report from the American Heart Association Statistics Committee and Stroke Statistics Subcommittee," Circulation, Vol. 119, No. 3, pp. 480486.

http://dx.doi.org/10.1161/CIRCULATIONAHA.108.1912 $\underline{59}$

[10] P. Schaapsmeerders, N. A. Maaijwee, E. J. van Dijk, L. C. Rutten-Jacobs, R. M. Arntz, H. C. Schoonderwaldt, L. D. Dorresteijn, R. P. Kessels and F. E. de Leeuw, "LongTerm Cognitive Impairment after First-Ever Ischemic Stroke in Young Adults,” Stroke, Vol. 44, No. 6, 2013, pp. 1621-1628.

http://dx.doi.org/10.1161/STROKEAHA.111.000792

[11] L. B. Goldstein, R. Adams, K. Becker, C. D. Furberg, P. B. Gorelick, G. Hademenos, M. Hill, G. Howard, V. J. Howard, B. Jacobs, S. R. Levine, L. Mosca, R. L. Sacco, D. G. Sherman, P. A. Wolf and G. J. del Zoppo, "Primary Prevention of Ischemic Stroke: A Statement for Healthcare Professionals from the Stroke Council of the American Heart Association,” Stroke, Vol. 32, No. 1, 2001, pp. 280-299. http://dx.doi.org/10.1161/01.STR.32.1.280

[12] G. L. Semenza, "HIF-1 and Human Disease: One Highly Involved Factor," Genes and Development, Vol. 14, Vol. 16, 2000, pp. 1983-1991.

[13] H. P. Adams Jr., B. H. Bendixen, L. J. Kappelle, J. Biller, B. B. Love, D. L. Gordon, E. E. Marsh 3"rd, "Classification of Subtype of Acute Ischemic Stroke. Definitions for Use in a Multicenter Clinical Trial. TOAST. Trial of Org 10172 in Acute Stroke Treatment,” Stroke, Vol. 24, No. 1, 1993, pp. 35-41. http://dx.doi.org/10.1161/01.STR.24.1.35

[14] D. I. Slater, S. A. Curtin, J. S. Johns, C. Schmidt, J. L. Tipton and R. Newbury, "Middle Cerebral Artery Stroke," 2008.

http://emedicine.medscape.com/article/ 323120-overview

[15] C. A. Anderson, D. B. Arciniegas, D. C. Huddle and M. 
A. Leehey, "Akinetic Mutism Following Unilateral Anterior Artery Occlusion,” Journal of Neuropsychiatry and Clinical Neuroscience, Vol. 15, No. 3, 2003, p. 3. http://dx.doi.org/10.1176/appi.neuropsych.15.3.385

[16] K. R. Bulsara, A. Zomorodi and J. M. Provenzale, “Anatomic Variant of the Posterior Cerebral Artery," American Journal of Roentgenology, Vol. 199, No. 4, 2007, p. W395. http://dx.doi.org/10.2214/AJR.06.0991

[17] E. K. Helseth, "Posterior Cerebral Artery Stroke,” 2012. http://emedicine.medscape.com/article/2128100-overview

[18] T. Pohjasvaara, T. Erkinjuntti, R. Ylikoski, M. Hietanen, R. Vataja and M. Kaste, "Clinical Determinants of Poststroke Dementia,” Stroke, Vol. 29, No. 1, 1998, pp. 7581. http://dx.doi.org/10.1161/01.STR.29.1.75

[19] H. Jokinen, H. Kalska, R. Mäntylä, T. Pohjasvaara , R. Ylikoski, M. Hietanen, O. Salonen, M. Kaste and T. Erkinjuntti, "Cognitive Profile of Subcortical Ischaemic Vascular Disease,” Journal of Neurology, Neurosurgery \& Psychiatry, Vol. 77, No. 1, 2006, pp. 28-33. http://dx.doi.org/10.1136/jnnp.2005.069120

[20] T. K. Tatemichi, D. W. Desmond, Y. Stern, M. Paik, M. Sano and E. Bagiella, "Cognitive Impairment after Stroke: Frequency, Patterns, and Relationship to Functional Abilities," Journal of Neurology, Neurosurgery \& Psychiatry, Vol. 57, No. 2, 1994, pp. 202-207.

http://dx.doi.org/10.1136/jnnp.57.2.202

[21] M. Hoffmann, "Higher Cortical Function Deficits after Stroke: An Analysis of 1,000 Patients from a Dedicated Cognitive Stroke Registry," Neurorehabilitation and Neural Repair, Vol. 15, No. 2, 2001, pp. 113-127. http://dx.doi.org/10.1177/154596830101500205

[22] G. A. Rosenberg, "Inflammation and White Matter Damage in Vascular Cognitive Impairment,” Stroke, Vol. 40, No. 3, 2009, pp. S20-S23. http://dx.doi.org/10.1161/STROKEAHA.108.533133

[23] G. Gold, E. Kövari, F. R. Herrmann, A. Canuto, P. R. Hof, J. P. Michel, C. Bouras and P. Giannakopoulos, "Cognitive Consequences of Thalamic, Basal Ganglia, and Deep White Matter Lacunes in Brain Aging and Dementia," Stroke, Vol. 36, No. 6, 2005, pp. 1184-1188. http://dx.doi.org/10.1161/01.STR.0000166052.89772.b5

[24] J. H. Garcia, "Stroke Pathophysiology, Diagnosis, and Management," In Barnett HJM Edition, Churchill Livingstone, New York, 1992.

[25] G. Goldenberg and C. Artner, "Visual Imagery and Knowledge about the Visual Appearance of Objects in Patients with Posterior Cerebral Artery Lesions," Brain and Cognition, Vol. 15, No. 2, 1991, pp. 60-86. http://dx.doi.org/10.1016/0278-2626(91)90024-3

[26] A. L. Georgiadis, Y. Yamamoto, E. S. Kwan, M. S. Pessin and L. R. Caplan, "Anatomy of Sensory Findings in Patients with Posterior Cerebral Artery Territory Infarction,” Archives in Neurology, Vol. 56, No. 7, 1999, pp. 835-838. http://dx.doi.org/10.1001/archneur.56.7.835

[27] D. Karussis, R. R. Leker and O. Abramsky, "Cognitive Dysfunction Following Thalamic Stroke: A Study of 16 Cases and Review of the Literature," Journal of Neurological Science, Vol. 172, No. 1, 2000, pp. 25-29. http://dx.doi.org/10.1016/S0022-510X(99)00267-1
[28] N. Gilberti, M. Gamba, A. Costa, V. Vergani, R. Spezi, A. Pezzini, I. Volonghi, D. Mardighian, R. Gasparotti, A. Padovani and M. Magoni, "Pure Midbrain Ischemia and Hypoplastic Vertebrobasilar Circulation,” Neurological Science, 2013. http://dx.doi.org/10.1007/s10072-013-1502-x

[29] P. Eslinger, "Neuropsychological Interventions: Clinical Research and Practice,” The Guilford Press, New York, 2005.

[30] A. Nitkunan, T. R. Barrick, R. A Charlton, C. A. Clark and H. S. Markus, "Multimodal MRI in Cerebral Small Vessel Disease: Its Relationship with Cognition and Sensitivity to Change over Time," Stroke, Vol. 39, No. 7, 2008, pp. 1999-2005. http://dx.doi.org/10.1161/STROKEAHA.107.507475

[31] M. O’Sullivan, R. G. Morris and H. S. Markus, "Brief Cognitive Assessment for Patients with Cerebral Small Vessel Disease," Journal of Neurology, Neurosurgery and Psychiatry, Vol. 76, No. 8, 2005, pp. 1140-1145. http://dx.doi.org/10.1136/jnnp.2004.045963

[32] G. Gainotti, M. Ferraccioli, M. G. Vita and C. Marra, "Patterns of Neuropsychological Impairment in MCI Patients with Small Subcortical Infarcts or Hippocampal atroPhy," Journal of the International Neuropsychology Society, Vol. 14, No. 4, 2008, pp. 611-619. http://dx.doi.org/10.1017/S1355617708080831

[33] M. Samuelsson, B. Söderfeldt and G. B. Olsson, "Functional Outcome in Patients with Lacunar Infarction,” Stroke, Vol. 27, No. 5, 1996, pp. 842-846. http://dx.doi.org/10.1161/01.STR.27.5.842

[34] T. Iwamoto, T. Shimizu, M. Ami, Y. Yoneda, T. Imamura and M. Takasaki, "Dementia and Disability after Initial Cerebral Thrombosis Evaluated by MRI and Their Clinical Course,” Abstract Nippon Ronen Igakkai Zasshi, Vol. 37, No. 2, 2000, pp. 62-69.

[35] B. T. Baune, A. Roesler, S. Knecht and K. Berger, "Single and Combined Effects of Cerebral White Matter Lesions and Lacunar Infarctions on Cognitive Function in an Elderly Population,” The Journals of Gerontology, Series A, Biological Sciences and Medical Sciences, Vol. 64, No. 1, 2009, pp. 118-124. http://dx.doi.org/10.1093/gerona/gln004

[36] J. S. Saczynski, S. Sigurdsson, M. K. Jonsdottir, G. Eiriksdottir, P. V. Jonsson, M. E. Garcia, O. Kjartansson, O. Lopez, M. A. van Buchem, V. Gudnason and L. J. Launer, "Cerebral Infarcts and Cognitive Performance: Importance of Location and Number of Infarcts," Stroke, Vol. 40, No. 3, 2009, pp. 677-682. http://dx.doi.org/10.1161/STROKEAHA.108.530212

[37] H. J. Wittsack, A. Ritzl, G. R. Fink, F. Wenserski, M. Siebler, R. J. Seitz, U. Mödder and H. J. Freund, "MR Imaging in Acute Stroke: Diffusion-Weighted and Perfusion Imaging Parameters for Predicting Infarct Size,” Radiology, Vol. 222, No. 2, 2002, pp. 397-403. http://dx.doi.org/10.1148/radiol.2222001731

[38] H. J. Aronen, M. P. Laakso, M. Moser and J. Perkiö, “Diffusion and Perfusion-Weighted Magnetic Resonance Imaging Techniques in Stroke Recovery,” Europa Medicophysica, Vol. 43, No. 2, 2007, pp. 271-284. 
[39] P. M. Rossini, C. Altamura, F. Ferreri, J. M. Melgari, F. Tecchio, M. Tombini, P. Pasqualetti and F. Vernieri, "Neuroimaging Experimental Studies on Brain Plasticity in Recovery from Stroke,” Europa Medicophysica, Vol. 43, No. 2, 2007, pp. 241-254.

[40] C. M. Bütefisch, R. Kleiser and R. J. Seitz, "Post-Lesional Cerebral Reorganisation: Evidence from Functional Neuroimaging and Transcranial Magnetic Stimulation,” Journal of Physiology-Paris, Vol. 99, No. 4-6, 2006, pp. 437454. http://dx.doi.org/10.1016/j.jphysparis.2006.03.001

[41] C. M. Bütefisch, "Plasticity in the Human Cerebral Cortex: Lessons from the Normal Brain and From Stroke," Neuroscientist, Vol. 10, No. 2, 2004, pp. 163-173. http://dx.doi.org/10.1177/1073858403262152

[42] C. D. Takahashi, L. Der Yeghiaian and S. C. Cramer, "Stroke Recovery and Its Imaging," Neuroimaging Clinics of North America, Vol. 15, No. 3, 2005, pp. 681-695. http://dx.doi.org/10.1016/j.nic.2005.08.006

[43] J. M. Mountz, H. G. Liu and G. Deutsch, "Neuroimaging in Cerebrovascular Disorders: Measurement of Cerebral Physiology after Stroke and Assessment of Stroke Recovery," Seminars in Nuclear Medicine, Vol. 33, No. 1, 2003, pp. 56-76. http://dx.doi.org/10.1053/snuc.2003.127293

[44] S. B. Ko and B. W. Yoon, "Mechanisms of Functional Recovery after Stroke,” Front Neurol Neurosci, Vol. 32, 2013, pp. 1-8. http://dx.doi.org/10.1159/000346405

[45] O. W. Witte, "Lesion-Induced Plasticity as a Potential Mechanism for Recovery and Rehabilitative Training," Current Opinion in Neurology, Vol. 11, No. 6, 1998, pp. 655662.

http://dx.doi.org/10.1097/00019052-199812000-00008

[46] O. W. Witte, I. Buchkremer-Ratzmann, K. Schiene, T. Neumann-Haefelin, G. Hagemann, M. Kraemer, K. Zilles and H. J. Freund, "Lesion-Induced Network Plasticity in Remote Brain Areas,” Trends in Neuroscience, Vol. 20, No. 8, 1997, pp. 348-349.

[47] J. B. Green, "Brain Reorganization after Stroke,” Topics in Stroke Rehabilitation, Vol. 10, No. 3, 2003, pp. 1-20. http://dx.doi.org/10.1310/H65X-23HW-QL1G-KTNQ

[48] S. H. Kreisel, H. Bäzner and M. G. Hennerici, "Pathophysiology of Stroke Rehabilitation: Temporal Aspects of Neurofunctional Recovery," Cerebrovascular Disease, Vol. 21, No. 1-2, 2006, pp. 6-17. http://dx.doi.org/10.1159/000089588

[49] P. M. Kidd, "Integrated Brain Restoration after Ischemic Stroke-Medical Management, Risk Factors, Nutrients, and Other Interventions for Managing Inflammation and Enhancing Brain Plasticity,” Alternative Medicine Review, Vol. 14, No. 1, 2009, pp. 14-35.

[50] N. S. Ward, M. M. Brown, A. J. Thompson and R. S. Frackowiak, "Neural Correlates of Outcome after Stroke: A Cross-Sectional fMRI Study,” Brain, Vol. 126, No. 6, 2003, pp. 1430-1448. http://dx.doi.org/10.1093/brain/awg145

[51] M. I. Posner, "Neural Systems and Individual Differences," Teachers College Record, Vol. 106, No. 1, 2003, pp. 24-30.

[52] P. Vitali, J. Abutalebi, M. Tettamanti, M. Danna, A. I.
Ansaldo, D. Perani, Y. Joanette and S. F. Cappa, “Training-Induced Brain Remapping in Chronic Aphasia: A Pilot Study," Neurorehabilitation and Neural Repair, Vol. 21, No. 2, 2007, pp. 152-160. http://dx.doi.org/10.1177/1545968306294735

[53] J. M. Mountz, "Nuclear Medicine in the Rehabilitative Treatment Evaluation in Stroke Recovery. Role of Dia= schisis Resolution and Cerebral Reorganization,” Europa Medicophysica, Vol. 43, No. 2, 2007, pp. 221-239.

[54] G. Nelles, "Cortical Reorganization-Effects of Intensive Therapy," Restorative Neurology and Neuroscience, Vol. 22, No. 3-5, 2004, pp. 239-244.

[55] P. M. Rossini and G. Dal Forno, "Integrated Technology for Evaluation of Brain Function and Neural Plasticity," Physical and Medical Rehabilitation Clinics of North America, Vol. 15, No. 1, 2004, pp. 263-306. http://dx.doi.org/10.1016/S1047-9651(03)00124-4

[56] N. S. Ward, "Future Perspectives in Functional Neuroimaging in Stroke Recovery,” Europa Medicophysica, Vol. 43, No. 2, 2007, pp. 285-294.

[57] T. Weiss, E. Hansen, L. Beyer, M. L. Conradi, F. Merten, C. Nichelmann, R. Rost and C. Zippel, "Activation Processes During Mental Practice in Stroke Patients,” International Journal of Psychophysiology, Vol. 17, No. 1, 1994, pp. 91-100.

http://dx.doi.org/10.1016/0167-8760(94)90059-0

[58] D. G. Nair, A. Fuchs, S. Burkart, F. L. Steinberg and J. A. S. Kelso, "Assessing Recovery in Middle Cerebral Artery Stroke Using Functional MRI,” Brain Injury, Vol. 19, No. 13, 2005, pp. 1165-1176. http://dx.doi.org/10.1080/02699050500149858

[59] P. Lindberg, H. Forssberg and J. Borg, "Rehabilitation after Stroke. Imaging Techniques Show How the Cortical Reorganization Is Affected by Training,” Lakartidningen, Vol. 100, No. 51-52, 2003, pp. 4289-4292.

[60] R. J. Seitz, C. M. Bütefisch, R. Kleiser and V. Hömberg, "Reorganisation of Cerebral Circuits in Human Ischemic Brain Disease,” Restorative Neurology and Neuroscience, Vol. 22, No. 3-5, 2004, pp. 207-229.

[61] S. L. Wolf, C. J. Winstein, J. P. Miller, E. Taub, G. Uswatte, D. Morris, C. Giuliani, K. E. Light, D. Nichols-Larsen and EXCITE Investigators, "Effect of Constraint-Induced Movement Therapy on Upper Extremity Function 3 to 9 Months after Stroke: The EXCITE Randomized Clinical Trial,” JAMA, Vol. 296, No. 17, 2006, pp. 2095-2104. http://dx.doi.org/10.1001/jama.296.17.2095

[62] B. H. Dobkin, "Functional MRI: A Potential Physiologic Indicator for Stroke Rehabilitation Interventions,” Stroke, Vol. 34, No. 5, 2003, pp. E26-E28. http://dx.doi.org/10.1161/01.STR.0000071140.00153.05

[63] M. V. Johnston, "Plasticity in the Developing Brain: Implications for Rehabilitation,” Developmental Disabilities Research Reviews, Vol. 15, No. 2, 2009, pp. 94-101. http://dx.doi.org/10.1002/ddrr.64

[64] F. Hamzei, J. Liepert, C. Dettmers, C. Weiller and M. Rijntjes, "Two Different Reorganization Patterns after Rehabilitative Therapy: An Exploratory Study with fMRI and TMS,” Neuroimage, Vol. 31, No. 2, 2006, pp. 710-720. 
http://dx.doi.org/10.1016/j.neuroimage.2005.12.035

[65] J. Liepert, F. Hamzei and C. Weiller, "Lesion-Induced and Training-Induced Brain Reorganization,” Restorative Neurology and Neuroscience, Vol. 22, No. 3-5, 2004, pp. 269-277.

[66] J. A. Brown, "Recovery of Motor Function after Stroke," Progress in Brain Research, Vol. 157, 2006, pp. 223- 228. http://dx.doi.org/10.1016/S0079-6123(06)57015-3

[67] T. Hodics, L. G. Cohen and S. C. Cramer, "Functional Imaging of Intervention Effects in Stroke Motor Rehabilitation," Archives of Physical and Medical Rehabilitation, Vol. 87, No. 12, 2006, pp. 36-42.

http://dx.doi.org/10.1016/j.apmr.2006.09.005

[68] H. S. Levin, "Neuroplasticity and Brain Imaging Research: Implications for Rehabilitation," Archives of Physical and Medical Rehabilitation, Vol. 87, No. 12, 2006, p. 1. http://dx.doi.org/10.1016/j.apmr.2006.09.010

[69] S. K. Schiemanck, G. Kwakkel, M. W. Post, L. J. Kappelle and A. J. Prevo, "Predicting Long-Term Independency in Activities of Daily Living after Middle Cerebral Artery Stroke: Does Information from MRI have Added Predictive Value Compared with Clinical Information?” Stroke, Vol. 37, No. 4, 2006, pp. 1050-1054. http://dx.doi.org/10.1161/01.STR.0000206462.09410.6f

[70] S. K. Schiemanck, G. Kwakkel, M. W. Post, L. J. Kappelle and A. J. Prevo, "Predictive Value of Ischemic Lesion Volume Assessed with Magnetic Resonance Imaging for Neurological Deficits and Functional Outcome Poststroke: A Critical Review of the Literature," Neurorehabilitation and Neural Repair, Vol. 20, No. 4, 2006, pp. 492-502. http://dx.doi.org/10.1177/1545968306289298

[71] M. A. Dimyan and L. G. Cohen, "Neuroplasticity in the Context of Motor Rehabilitation after Stroke," Nature Reviews Neurology, Vol. 7, No. 2, 2011, pp. 76-85.

[72] L. Snaphaan, M. Rijpkema, I. van Uden, G. Fernández and F. E. de Leeuw, "Reduced Medial Temporal Lobe Functionality in Stroke Patients: A Functional Magnetic Resonance Imaging Study,” Brain, Vol. 132, No. 7, 2009, pp. 1882-1888. http://dx.doi.org/10.1093/brain/awp133

[73] E. A. Schouten, S. K. Schiemanck, N. Brand and M. W. Post, "Long-Term Deficits in Episodic Memory after Ischemic Stroke: Evaluation and Prediction of Verbal and Visual Memory Performance Based on Lesion Characteristics," Journal of Stroke and Cerebrovascular Disease, Vol. 18, No. 2, 2009, pp. 128-138. http://dx.doi.org/10.1016/j.jstrokecerebrovasdis.2008.09.0 $\underline{17}$

[74] O. Godefroy, M. Roussel, X. Leclerc and D. Leys, "Deficit of Episodic Memory: Anatomy and Related Patterns in Stroke Patients," European Neurology, Vol. 61, No. 4, 2009, pp. 223-229. http://dx.doi.org/10.1159/000197107

[75] R. S. Ross and S. D. Slotnick, “The Hippocampus Is Preferentially Associated with Memory for Spatial Context," Journal of Cognitive Neuroscience, Vol. 20, No. 3, 2008, pp. 432-446. http://dx.doi.org/10.1162/jocn.2008.20035

[76] B. C. Dickerson, D. H. Salat, J. F. Bates, M. Atiya, R. J.
Killiany, D. N. Greve, A. M. Dale, C. E. Stern, D. Blacker, M. S. Albert and R. A. Sperling, "Medial Temporal Lobe Function and Structure in Mild Cognitive Impairment," Annals of Neurology, Vol. 56, No. 1, 2004, pp. 27-35. http://dx.doi.org/10.1002/ana.20163

[77] G. S. Harrington, S. Tomaszewski Farias, M. H. Buonocore and A. P. Yonelinas, “The Intersubject and Intrasubject Reproducibility of FMRI Activation during Three Encoding Tasks: Implications for Clinical Applications," Neuroradiology, Vol. 48, No. 7, 2006, pp. 495-505. http://dx.doi.org/10.1007/s00234-006-0083-2

[78] C. Windischberger, C. Lamm, H. Bauer and E. Moser, "Consistency of Inter-Trial Activation Using Single-Trial fMRI: Assessment of Regional Differences,” Cognitive Brain Research, Vol. 13, No. 1, 2002, pp. 129-138. http://dx.doi.org/10.1016/S0926-6410(01)00101-X

[79] H. Koga, Y. Takashima, R. Murakawa, A. Uchino, T. Yuzuriha and H. Yao, "Cognitive Consequences of Multiple Lacunes and Leukoaraiosis as Vascular Cognitive Impairment in Community-Dwelling Elderly Individuals," Journal of Stroke and Cerebrovascular Disease, Vol. 18, No. 1, 2009, pp. 32-37. http://dx.doi.org/10.1016/j.jstrokecerebrovasdis.2008.07.0 $\underline{10}$

[80] L. C. Gioia, É. Tollard, V. Dubuc, S. Lanthier, Y. Deschaintre, M. Chagnon and A.Y. Poppe, "Silent Ischemic Lesions in Young Adults with First Stroke Are Associated with Recurrent Stroke,” Neurology, Vol. 79, No. 12, 2012, pp. 1208-1214. http://dx.doi.org/10.1212/WNL.0b013e31826aacac

[81] B. Levine and M. M. Downey-Lamb, "Design and Evaluation of Rehabilitation Experiments," In: P. Eslinger, Ed., Neuropsychological Interventions: Clinical Research and Practice, The Guilford Press, New York, 2005, pp. 80-102.

[82] T. Manly, S. Ward and I. Robertson, "The Rehabilitation of Attention,” In: P. Eslinger, Ed., Neuropsychological Interventions: Clinical Research and Practice, The Guilford Press, New York, 2005, pp. 105-136.

[83] K. D. Cicerone, “Remediation of 'Working Attention' in Mild Traumatic Brain Injury,” Brain Injury, Vol. 16, No. 3, 2002, pp. 185-195. http://dx.doi.org/10.1080/02699050110103959

[84] K. D. Cicerone, C. Dahlberg, K. Kalmar, D. M. Langenbahn, J. F. Malec, T. F. Bergquist, T. Felicetti, J. T. Giacino, J. P. Harley, D. E. Harrington, J. Herzog, S. Kneipp, L. Laatsch and P. A. Morse, "Evidence-Based Cognitive Rehabilitation: Recommendations for Clinical Practice,” Archives of Physical and Medical Rehabilitation, Vol. 81, No. 12, 2000, pp. 1596-1615. http://dx.doi.org/10.1053/apmr.2000.19240

[85] K. D. Cicerone, C. Dahlberg, J. F. Malec, D. M. Langenbahn, T. Felicetti, S. Kneipp, W. Ellmo, K. Kalmar, J. T. Giacino, J. P. Harley, L. Laatsch, P. A. Morse and J. Catanese, "Evidence-Based Cognitive Rehabilitation: Updated Review of the Literature from 1998 through 2002," Archives of Physical and Medical Rehabilitation, Vol. 86, No. 8, 2005, pp. 1681-1692. http://dx.doi.org/10.1016/j.apmr.2005.03.024 
[86] M. Reuter, U. Ott, D. Vaitl and J. Hennig, "Impaired Executive Control Is Associated with a Variation in the Promoter Region of the Tryptophan Hydroxylase 2 Gene," Journal of Cognitive Neuroscience, Vol. 19, No. 3, 2007, pp. 401-408. http://dx.doi.org/10.1162/jocn.2007.19.3.401

[87] P. Malhotra, E. J. Coulthard and M. Husain, "Role of Right Posterior Parietal Cortex in Maintaining Attention to Spatial Locations over Time,” Brain, Vol. 132, No. 3, 2009, pp. 645-660.

http://dx.doi.org/10.1093/brain/awn350

[88] J. O’Shea, “Cognitive Neurology: Stimulating Research on Neglect," Current Biology, Vol. 19, No. 2, 2009, pp. R76-R78. http://dx.doi.org/10.1016/j.cub.2008.11.038

[89] M. Thimm, G. R. Fink, J. Küst, H. Karbe and W. Sturm, "Impact of Alertness Training on Spatial Neglect: A Behavioural and fMRI Study," Neuropsychologia, Vol. 44, No. 7, 2006, pp. 1230-1246. http://dx.doi.org/10.1016/j.neuropsychologia.2005.09.008

[90] M. Thimm, G. R. Fink and W. Sturm, "Neural Correlates of Recovery from Acute Hemispatial Neglect," Restorative Neurology and Neuroscience, Vol. 26, No. 6, 2008, pp. 481-492.

[91] R. E. Hanlon, B. H. Dobkin, B. Hadler, S. Ramirez and Y. Cheska, "Neurorehabilitation Following Right Thalamic Infarct: Effects of Cognitive Retraining on Functional Performance," Journal of Clinical and Experimental Neuropsychology, Vol. 14, No. 4, 1992, pp. 433-447. http://dx.doi.org/10.1080/01688639208402836

[92] Y. H. Kim, W. K. Yoo, M. H. Ko, C. H. Park, S. T. Kim and D. L. Na, "Plasticity of the Attentional Network after Brain Injury and Cognitive Rehabilitation,” Neurorehabilitation and Neural Repair, Vol. 23, No. 5, 2009, pp. 468-477. http://dx.doi.org/10.1177/1545968308328728

[93] C. A. Hanlon, A. L. Buffington and M. J. McKeown, "New Brain Networks Are Active after Right MCA Stroke When Moving the Ipsilesional Arm," Neurology, Vol. 64, No. 1, 2005, pp. 114-120. http://dx.doi.org/10.1212/01.WNL.0000148726.45458.A9

[94] B. H. Dobkin, "Neurobiology of Rehabilitation,” Annals of New York Academy of Science, Vol. 1038, 2004, pp. 148-170. http://dx.doi.org/10.1196/annals.1315.024

[95] D. Perani, S. F. Cappa, M. Tettamanti, M. Rosa, P. Scifo, A. Miozzo, A. Basso and F. Fazio, "A fMRI Study of Word Retrieval in Aphasia,” Brain and Language, Vol. 85, No. 3, 2003, pp. 357-368. http://dx.doi.org/10.1016/S0093-934X(02)00561-8

[96] Y. Cao, K. P. George, J. R. Ewing, E. M. Vikingstad and A. F. Johnson, "Neuroimaging of Language and Aphasia after Stroke," Journal of Stroke and Cerebrovascular Disease, Vol. 7, No. 4, 1998, pp. 230-233. http://dx.doi.org/10.1016/S1052-3057(98)80031-1

[97] S. H. Yee, H. L. Liu, J. W. Hou, Y. L. Pu, P. T. Fox and J. H. Gao, "Detection of the Brain Response during a Cog- nitive Task Using Perfusion-Based Event-Related Functional MRI,” Neuroreport, Vol. 11, No. 11, 2000, pp. 25332536. http://dx.doi.org/10.1097/00001756-200008030-00037

[98] G. A. Calvert, M. J. Brammer, R. G. Morris, S. C. Williams, N. King and P. M. Matthews, "Using fMRI to Study Recovery from Acquired Dysphasia,” Brain and Language, Vol. 71, No. 3, 2000, pp. 391-399. http://dx.doi.org/10.1006/brln.1999.2272

[99] J. Fridriksson, L. Morrow-Odom, D. Moser, A. Fridriksson and G. Baylis, "Neural Recruitment Associated with Anomia Treatment in Aphasia,” NeuroImage, Vol. 32, No. 3, 2006, pp. 1403-1412. http://dx.doi.org/10.1016/j.neuroimage.2006.04.194

[100] M. Meinzer, T. Flaisch, C. Breitenstein, C. Wienbruch, T. Elbert and B. Rockstroh, "Functional Re-Recruitment of Dysfunctional Brain Areas Predicts Language Recovery in Chronic Aphasia,” NeuroImage, Vol. 39, No. 4, 2008, pp. 2038-2046. http://dx.doi.org/10.1016/j.neuroimage.2007.10.008

[101] B. Fernandez, D. Cardebat, J. F. Demonet, P. A. Joseph, J. M. Mazaux, M. Barat and M. Allard, "Functional MRI Follow-Up Study of Language Processes in Healthy Subjects and during Recovery in a Case of Aphasia," Stroke, Vol. 35, No. 9, 2004, pp. 2171-2176. http://dx.doi.org/10.1161/01.STR.0000139323.76769.b0

[102] S. K. Bhogal, R. Teasell and M. Speechley, "Intensity of Aphasia Therapy, Impact on Recovery,” Stroke, Vol. 34, No. 4, 2003, pp. 987-993.

http://dx.doi.org/10.1161/01.STR.0000062343.64383.D0

[103] J. Kuest and H. Karbe, "Cortical Activation Studies in Aphasia,” Current Neurology and Neuroscience Reports, Vol. 2, No. 6, 2002, pp. 511-515. http://dx.doi.org/10.1007/s11910-002-0038-x

[104] R. W. easell, “Stroke Recovery and Rehabilitation,” Stroke, Vol. 34, 2003, pp. 365-366. http://dx.doi.org/10.1161/01.STR.0000054630.33395.E2

[105] A. Nascimbeni, A. Gaffuri and P. Imazio, "Motor Evoked Potentials: Prognostic Value in Motor Recovery after Stroke,” Functional Neurology, Vol. 21, No. 4, 2006, pp. 199-203.

[106] J. A. Detre and T. F. Floyd, "Functional MRI and Its Applications to the Clinical Neurosciences," Neuroscientist, Vol. 7, No. 1, 2001, pp. 64-79. http://dx.doi.org/10.1177/107385840100700110

[107] S. G. Romero, C. F. Manly and J. Grafman, "Investigating Cognitive Neuroplasticity in Single Cases: Lessons Learned from Applying Functional Neuroimaging Techniques to the Traditional Neuropsychological Case Study Framework," Neurocase, Vol. 8, No. 5, 2002, pp. 355368. http://dx.doi.org/10.1076/neur.8.4.355.16187 